\title{
Análisis psicodinámico breve en un caso de dependencia del alcohol.
}

Brief Psychodynamic analysis in a case of alcohol dependence.

\begin{abstract}
Benjamín Cortés Morales a , Alejandro Ballesteros Prados ${ }^{\text {b }}$, Joemir Becker Costa ${ }^{\mathrm{c}}$.
${ }^{a}$ Psiquiatra y Máster en psicoterapia. Clínica de Psiquiatría y Psicoterapia, Dr. B. Cortés. Salamanca, España. ${ }^{b}$ Psiquiatra. Moorside Unit, Trafford General Hospital. Manchester, United Kingdom. ${ }^{c}$ Psiquiatra y Máster en Psicoterapia. Servicio de Psiquiatría. Sección de Medicina Psicosomática. Hospital Universitario de Salamanca. Salamanca, España.
\end{abstract}

Correspondencia: Benjamín Cortés Morales (consulta@drbcortes.com)

Recibido: 27/01/2013; aceptado: 12/06/2013

RESUMEN: Se presenta el caso de un paciente masculino de 41 años de edad diagnosticado con dependencia del alcohol, con el objetivo de elaborar un juicio diagnóstico y pronóstico desde el punto de vista psicodinámico, y descubrir, en la medida de lo posible, "quién" es el paciente, qué desarrollo vital le ha llevado a ser lo que actualmente es y derivando de esta concepción, qué sentido tiene su "enfermar".

PALABRAS CLAVE: alcoholismo, psicoanálisis, diagnóstico, psicología clínica.
ABSTRACT: We report a case of a client diagnosed of alcohol dependence with the aim of developing a psychodynamic approach to study his diagnosis and prognosis. In addition, we pretend to address "who" is the patient, the biographic development that led him to have his current "self" and, in relation with this concept, to what extent his mental disorder is related with the whole biographical picture.

KEY WORDS: Alcoholism, Psychoanalysis, diagnosis, clinical psychology.

\section{Introducción}

Toda metodología exploratoria en psiquiatría presupone en sí misma una concepción de la enfermedad y la propia concepción de lo que ha de ser la terapia. La Historia Clínica Psiquiátrica permite establecer una nosotaxia (a nivel de síntomas, síndromes o trastornos) que a su vez permite elegir los psicofármacos adecuados. A diferencia de esta, la Historia Clínica Psicodinámica, desde un punto de vista neopsicoanalítico, está concebida para 1) agilizar la obtención del material durante la fase de exploración, así como para la sistematización de la información para llegar a un diagnóstico clínico y dinámico, 2) determinar en lo posible la elaboración de una hipótesis inicial de trabajo dirigido al insight, y 3) permitir establecer un pronóstico y los posibles límites de la terapia psicodinámica. En este artículo se presenta el caso de un paciente dependiente del alcohol con el objetivo de elaborar un juicio diagnóstico y pronóstico desde el punto de vista psicodinámico y descubrir en la medida de lo posible, "quién" es el paciente, qué desarrollo vital le ha llevado a ser lo que actualmente es y derivando de esta concepción, qué sentido tiene su "enfermar". La actitud exploratoria del entrevistador durante los cuatro encuentros de dos horas cada uno con el paciente fue de "comprensión" del material advertido (revisión biográfica del sujeto, del ambiente creado por el mismo y el encuadre 
familiar actual), y del material inadvertido (recuerdos infantiles, los sueños y las ilusiones y fantasías), y no una "interpretación" de los mismos (1).

\section{Anamnesis}

Varón de 41 años de edad, separado, vive actualmente con sus padres aunque pasa temporadas viviendo en casa de sus hermanos. Estudió hasta tercero de "Enseñanza General Básica" por motivos económicos, es oficial de panadero, actualmente sin trabajo y recibe una pensión del estado. Ha sido diagnosticado seropositivo al VIH, VHB y VHC, sin tratamiento. Hepatopatía alcohólica, hipoacusia neurosensorial bilateral por trauma acústico en la infancia. Antecedentes de consumo de cannabis, cocaína y heroína, actualmente inactivo. Alcoholismo y tabaquismo desde la juventud.

Dos ingresos anteriores en la Unidad de Hospitalización Breve en los últimos cuatro años, por ideación autolítica y dependencia al alcohol. Estuvo ingresado en Proyecto Hombre por cuatro años tras lo cual abandonó el uso de la cannabis, cocaína y heroína, sin embargo ha continuado consumiendo alcohol en grandes cantidades hasta la actualidad. En el ingreso actual, el paciente acude a urgencia voluntariamente después de haber realizado gesto autolítico con 40 comprimidos de cotrimoxazol. Presentaba una clínica de corte depresiva, que incluía ideas puntuales de muerte que relaciona con la recaída actual en el consumo de alcohol.

\section{Estudio psicodinámico}

1. Relación paciente-examinador. Forma global de la relación de objeto:

La forma global de relación del paciente con los objetos se refiere a la forma de comunicación del paciente con el examinador (patrón que suele repetir con otras figuras de autoridad), esta se estudia en la relación transferencial y contratransferencial. En este paciente se observa una relación de tipo mágica, la cual se expresa desde las primeras entrevistas a través de la admiración por las figuras de autoridad y espera pacientemente a que "le curen" ("estoy aquí para que me ayuden, quiero curarme"). En ocasiones toma el papel "Dependiente", en la que muestra una actitud de obediencia y eficacia, atendiendo todo, tratando de captar todo. De igual manera, la forma básica predominante en su vida ha sido la "mágica", ha aprendido a idealizar a las figuras de autoridad; su padre (alcohólico, como una gran cantidad de tíos y primos) falleció por infarto agudo del miocardio cuando el paciente tenía 
NOTAS CLÍNICAS

11 años de edad. El paciente recuerda a su padre como una persona maravillosa, buen trabajador, alguien a quién hay que imitar. Para él, su madre es la persona más abnegada que conoce, con una admiración y sumisión extrema. Su hermano mayor es el encargado de suministrar el dinero al paciente de forma ocasional para sus gastos. Depende de la pensión del estado para vivir.

\section{Impresión contratransferencial del examinador:}

El movimiento emocional que me evoca el paciente tiene dos caras. Por un lado me provoca lástima, pena e intención de ayudarle; sin embargo, por otro lado me es difícil imaginarlo sano, difícilmente capto sus aspectos adultos y me es fácil verlo enfermo. La escena temida es que me sobrepase el desánimo, la impaciencia y yoquepierdismo debido a las continuas recaídas.

\section{Juicio diagnóstico (2):}

Estructura de la personalidad: tendencias predominantes.

En psicoanálisis la estructura de carácter representa la forma particular en la cual la energía está encausada en el proceso de vivir. En la doctrina psicoanalítica freudiana el carácter responde al tipo de organización de la libido (oral, anal, genital), mientras que la doctrina neoanalítica considera como base fundamental los modos específicos de relación de la persona con el mundo (3), es decir sus tendencias predominantes (orientaciones receptiva, explotadora, acumulativa, mercantil, productiva). El caso del paciente responde a una orientación receptiva, este suele esperar pasivamente de las figuras de autoridad los suministros para vivir (dinero, alimentos, casa, e incluso la droga); no suele discriminar lo que introyecta, todas sus relaciones sexuales han sido con parejas en las que la probabilidad de alcanzar una relación estable es muy difícil (mujeres que se dedican a la prostitución); en general suele ser cordial, sumiso y obediente con "los que lo alimentan" y se conforma con lo poco que le den. El carácter también responde ocasionalmente a una orientación explotadora, desear la propiedad de otro; su historia delictiva consiste en innumerables atracos, robos con y sin violencia aunque solo haya sido detenido una vez por la policía, quien luego lo dejó en libertar para ingresar a un tratamiento rehabilitador en Proyecto Hombre; el paciente suele ser desconfiado, por lo que se mantiene en todo momento a la defensiva y puede ser muy agresivo cuando se encuentra acorralado, este casi mata a una persona en uno de sus atracos; como aspecto positivo se puede decir que es capaz de reclamar cuando se siente agredido y de tomar la iniciativa aunque muchas veces precipitada.

Estructura de la personalidad: defensa predominante (4).

El concepto de defensa predominante tiene más que ver con las estructuras de "resistencia" que de defensa misma. Más que nombrar las defensas del yo se obser- 
van "las técnicas sistemáticas de defensa y evitación" mediante las cuales el enfermo trata de mantener la distancia adecuada para evitar la movilización del núcleo "psicótico" de base (ver más adelante). El paciente muestra principalmente dos tipos de defensas, 1) la técnica de externalización: este descarga la tensión emocional por medio de la acción, por ejemplo los activos delictivos, el robo, la violencia (desde niño intimidaba a otros niños para obtener su ropa nueva o cualquier otra pertenencia), consumo de drogas sin discriminar los medios de administración de estas; 2) la técnica demostrativa: los intentos de satisfacción son logrados mediante la manipulación en su familia, los símbolos idealizados, son tratados como reales, por ejemplo su padre es idealizado, cuando en realidad este fue un alcohólico, que profundizó la pobreza en la familia, al punto que todos los hijos trabajaron desde la infancia (en el caso de nuestro paciente desde los cuatro años). Entre los mecanismos de defensa se observa la imposición como medio de manejo de otros, la amenaza de otras personas para poder conseguir los tóxicos, incluyendo el alcohol, que se convirtió en el diario vivir.

Estructura de la personalidad: núcleo psicótico de base.

Desde las aportaciones de la escuela psicoanalítica argentina y de las concepciones Kleinianas introducidas por Pichón-Rivière (5), se entiende que a partir de los núcleos psicóticos de base intuidos por la historia clínica dinámica, se expresan las defensas predominantes vistas anteriormente, es decir la conducta del sujeto. El concepto "núcleos psicóticos" se debe entender como "núcleos ansiosos", de tal manera que las personas reaccionan de una manera determinada para evitar el tipo de núcleo ansioso que manifiesta. Existen tres núcleos psicóticos: el confusional, el paranoide y el depresivo. En el paciente se expresa un núcleo confusional, este se intuye por una superestructura en las tres áreas principales: 1) en el área de la mente predomina el sentimiento de indiscriminación (no discrimina) y patrones para valorar si las amenazas se generan en el interior de sí mismo o provienen del mundo externo, el paciente no solo expresa violencia hacia los objetos sino que ha planeado e intentado suicidarse en cuatro ocasiones; 2) en el área de la corporalidad, la ansiedad de búsquedas de sensaciones (sexo con prostitutas, incorporación de drogas, el juego) son difícilmente sistematizables; 3 ) en el área de las relaciones interpersonales el paciente no define bien quienes son protectores y quienes hacen daño, desde pequeño ha sido explotado por personas que ahora considera "buenas personas", estos han sido narcotraficantes que le daban la droga gratis para "engancharlo", trabajos muy mal remunerados, incluso personas que le han encargado atracos de gran riesgo.

Estructura de la personalidad: tendencias progresivas y regresvas:

Las tendencias progresivas y regresivas son conceptos desarrollados por Erich Fromm (6), se dividen en aquellas que llevan a la decadencia y aquellas que 
NOTAS CLÍNICAS

llevan al crecimiento. En el paciente se encuentra de forma clara un síndrome de decadencia, compuesto por la necrofilia y la simbiosis incestuosa. En el paciente, la necrofilia se expresa en el gusto de hablar de sus desgracias y sus enfermedades, toda su alegría está en el pasado infantil (“fui un niño muy feliz"), es frío en sus relaciones. La simbiosis incestuosa (no entendida como incestos, sino como la presencia relaciones interpersonales con límites difusos) se expresa en la necesidad de una figura maternizante con la que contar incondicionalmente, es un buscador permanente de gratificación.

\section{Estructura identificativa y grado de individuación:}

La estructura identificativa de una persona es la forma que tiene de identificarse con el otro, qué tan autónomo es el paciente en sus relaciones con las otras personas. En el caso que nos ocupa, el paciente muestra una identificación regresiva, es decir la continua reproducción de situaciones identificativas en un estado de unión/nodiferencia, como la utiliza un niño con sus progenitores o cuidadores, donde él es el todo y los demás forman parte de él, es decir sus vidas están a su servicio.

\section{Discusión}

La discusión en forma de síntesis del caso responde a las siguientes preguntas: 1) ¿Cuál es la significación específica de la "causa" desencadenante en relación con la técnica defensiva propia del sujeto? La causa desencadentante ha sido la incapacidad a lo largo de la vida de interiorizar los eventos vitales (muerte, fracaso de sus relaciones de pareja, pérdida de la salud por enfermedades graves, intentos autolíticos) y darles salida por medio de defensas maduras. 2) ¿Por qué no se ha podido reestructurar la defensa? La defensa no se reestructura por que la necesita, necesita de estas defensas inmaduras para su sobrevivencia en el medio que vive y el medio que ha sido creado por comportamiento, necesita estar enfermo para conseguir dinero, comida (dependencia de familiares y del estado) y necesidades de aceptación, adopción y amor. 3) ¿Ha habido una modificación de base de las circunstancias y de las demandas existenciales del sujeto? No, las demandas existenciales del sujeto no han sido satisfechas como son la descendencia ("añoro un hijo"), una pareja estable, constancia y estabilidad ("no estar dando tumbos"). 4) ¿Está la enfermedad llenando una misión específica en el sujeto? Sin duda llena una misión y es el generar compasión. 5) ¿De qué situación temida defiende la enfermedad? La enfermedad le defiende de perder sus beneficios como son personas que se preocupan por él, gente que le da de comer, vivir de la caridad y los beneficios de las instituciones a las cuales puede acudir en caso de desventura. 


\section{BIBLIOGRAFIA:}

(1) Fromm E. El lenguaje olvidado: Introducción a la comprensión de los sueños, mitos y cuentos de hadas. Buenos Aires: Editorial Hachette; 1972.

(2) Menninger KA. A Manual for Psychiatric Case Study. New York: Grune \& Stratton; 1952.

(3) Fromm E. Ética y psicoanálisis. México: Fondo de Cultura; 1973.

(4) Reich W. Análisis de carácter. Buenos Aires: Paidós Ibérica; 2005.

(5) Pichón Rivière E. Teoría del vínculo. Buenos Aires: Nueva Visión, Buenos Aires; 1980.

(6) Fromm E. El corazón del hombre. Madrid: Fondo de cultura económica de España, 1980. 